\title{
Efecto de Saccharomyces cerevisiae y tiempos de fermentación sobre la composición química del ensilado de avena y cebada
}

\author{
Effect of Saccharomyces cerevisiae and fermentation times on the chemical \\ composition of oat and barley silage
}

\begin{abstract}
Ricardo Fernandez Taype ${ }^{1}$, Jose Luis Contreras Paco ${ }^{2,4}$, James Curasma Ccente ${ }^{1}$, Alfonso Cordero Fernández ${ }^{3}$, Yhan Carlos Rojas De La Cruz ${ }^{1}$, David Ruiz Vilchez ${ }^{2}$, Rodrigo Huaman Jurado ${ }^{3}$
\end{abstract}

\section{Resumen}

\begin{abstract}
El objetivo del estudio fue evaluar los efectos de la levadura Saccharomyces cerevisiae como aditivo para la preparación de ensilado de avena y cebada en dos tiempos de fermentación ( 21 y 42 días). Se prepararon minisilos de avena y cebada y se evaluaron cuatro niveles de levadura $(0,15,30$ y $45 \mathrm{~g} / \mathrm{kg} \mathrm{FV})$. Los minisilos fueron abiertos a los 21 y 42 días. Se prepararon tres repeticiones de ensilado para cada combinación de ensilado $\mathrm{x}$ nivel de levadura $\mathrm{x}$ tiempo de ensilado. Los valores de composición bromatológica del ensilado de avena en términos de proteína cruda (PC), materia mineral $(\mathrm{MM})$, fibra detergente neutra (FDN), fibra detergente ácida (FDA), extracto etéreo (EE) y $\mathrm{pH}$ aumentaron cuando se agregó la levadura con relación al control, pero disminuyó la materia seca (MS). La calidad del ensilado de cebada en términos de MS, PC, FDN, FDA y EE mejoró al agregar 30 y 45 g/kg de levadura, en comparación al control; sin embargo, disminuyó para EE y pH. Los resultados demuestran que la inoculación de S. cerevisiae
\end{abstract}

\footnotetext{
${ }^{1}$ Laboratorio de Nutrición Animal y Evaluación de Alimentos, Universidad Nacional de Huancavelica, Huancavelica, Perú

${ }^{2}$ Facultad de Ciencias Agrarias, Universidad Nacional de Huancavelica, Huancavelica, Perú

${ }^{3}$ Facultad de Ciencias de Ingeniería, Universidad Nacional de Huancavelica, Huancavelica, Perú

${ }^{4}$ Email.jose.contreras@unh.edu.pe
}

Recibido: 2 de marzo de 2021

Aceptado para publicación: 5 de noviembre de 2021

Publicado: 22 de diciembre de 2021

CLos autores. Este artículo es publicado por la Rev Inv Vet Perú de la Facultad de Medicina Veterinaria, Universidad Nacional Mayor de San Marcos. Este es un artículo de acceso abierto, distribuido bajo los términos de la licencia Creative Commons Atribución 4.0 Internacional (CC BY 4.0) [https:// creativecommons.org/licenses/by/4.0/deed.es] que permite el uso, distribución y reproducción en cualquier medio, siempre que la obra original sea debidamente citada de su fuente original 
en los dos tiempos de fermentación incrementa el contenido de PC, EE, MM y pH del ensilado; sin embargo; el alto nivel de $\mathrm{pH}$ no permite conservar la calidad del ensilado. Se concluye que el mejor valor nutricional se obtuvo con la adición de $S$. cerevisiae de $45 \mathrm{~g} /$ $\mathrm{kg}$ a los 42 días de fermentación en ambos ensilados, aunque disminuye algunos parámetros de importancia.

Palabras clave: levadura, silo, valor nutritivo

\section{Abstract}

The aim of this study was to evaluate the effects of the yeast Saccharomyces cerevisiae as an additive for the preparation of oat and barley silage in two fermentation times (21 and 42 days). Oat and barley minisilos were prepared using four levels of yeast $(0,15,30$ and 45 $\mathrm{g} / \mathrm{kg} \mathrm{FV).} \mathrm{The} \mathrm{minisilos} \mathrm{were} \mathrm{opened} \mathrm{at} 21$ and 42 days. Three silage replicates were prepared for each combination of silage $\mathrm{x}$ yeast level $\mathrm{x}$ silage time. The bromatological composition values of the oat silage in terms of crude protein (CP), mineral matter (MM), neutral detergent fibre (NDF), acid detergent fibre (FDA), ethereal extract (EE) and $\mathrm{pH}$ increased when the yeast was added in relation to the control, but the dry matter (DM) decreased. The quality of the barley silage in terms of DM, CP, NDF, FDAand EE improved when adding 30 and $45 \mathrm{~g} / \mathrm{kg}$ of yeast, compared to the control; however, it decreased for $\mathrm{EE}$ and $\mathrm{pH}$. The results show that the inoculation of $S$. cerevisiae in the two fermentation times increases the content of CP, EE, MM and $\mathrm{pH}$ of the silage; nevertheless; the high $\mathrm{pH}$ level does not allow proper conservation of the quality of the silage. It is concluded that the best nutritional values were obtained with the addition of $S$. cerevisiae of $45 \mathrm{~g} / \mathrm{kg}$ at 42 days of fermentation in both silages, although it decreases some important parameters.

Key words: yeast, silo, nutritional value

\section{INTRODUCCIÓN}

En el Perú, los cultivos forrajeros más abundantes son las gramíneas forrajeras como avena (Avena sativa), cebada (Hordeum vulgare), triticale ( $x$ Triticosecale) y rye grass (Lolium perenne), los cuales se vienen sembrando con desconocimiento sobre sus ventajas agronómicas y nutricionales por parte de los pequeños productores (Ordoñez y Bojórquez, 2011). El ganado es alimentado con recursos forrajeros de baja calidad nutricional, especialmente durante el periodo seco, trayendo como consecuencia bajos niveles de producción y productividad pecuaria (Roque, 2012).
El ensilado es un método eficaz para reducir la pérdida de nutrientes durante el almacenamiento y conservar el forraje fresco para un uso prolongado (Zhou et al., 2019). El uso de aditivos en el ensilado se ha centrado principalmente con fines de mejorar la calidad de la fermentación durante el sellado y la estabilidad aeróbica durante la fase de alimentación (Muck et al., 2018). En la práctica, la demanda actual de los aditivos o inoculantes de ensilaje tiene por finalidad mejorar la calidad nutricional y regular la microflora intestinal (Zhou et al., 2019). Saccharomyces cerevisiae es un microorganismo ampliamente utilizado (Shurson, 2018), dado que ayuda a modular el sistema inmunológico de los animales jóvenes 
(Trckova et al., 2014; Broadway et al., 2015), mejorando la fermentación del rumen (Zhu et al., 2017) y la degradabilidad de los nutrientes del forraje en el intestino (Elghandour et al., 2016).

Además, al incluir estos aditivos en la alimentación de los rumiantes, ciertas levaduras han demostrado aumentar la población de bacterias celulolíticas y mejorar la tasa de crecimiento y la producción de leche, así como eliminar patógenos del tracto intestinal (McAllister et al., 2011), lo que sería una ventaja adicional de usar levaduras como inoculante de ensilaje si esos microorganismos son capaces de resistir el ensilado. Por lo tanto, el objetivo de este estudio fue evaluar el valor nutricional del ensilado de avena y cebada con diferentes niveles de $S$. cerevisiae en dos tiempos de fermentación.

\section{Materiales y Métodos}

El estudio se ejecutó en las instalaciones de la Escuela Profesional de Zootecnia y en el Laboratorio de nutrición y evaluación de alimentos (LUNEA) de la Facultad de Ciencias de Ingeniería de la Universidad Nacional de Huancavelica, ubicados en la localidad de Paturpampa, Huancavelica, Perú, a una altitud de $3760 \mathrm{~m}$. Se utilizó avena (Avena sativa) variedad Mantaro 15 y cebada variedad UNA 80 cosechadas en etapa de grano lechoso, traídos directamente del campo de la comunidad de Antaccocha, Huancavelica. Estos forrajes fueron sembrados en noviembre de 2018 y cosechados en abril de 2019.

Se obtuvo $36 \mathrm{~kg}$ de forraje verde (FV) de avena y cebada para el proceso del ensilado picándose con una máquina picadora de forraje de tres cuchillas Maquiagro con tamaños aproximados de picado de 2.5 a 3.5 $\mathrm{cm}$. Luego se incorporó la levadura (Saccharomyces cerevisiae) en niveles de $0,1.5,3$ y $4.5 \%$ en 48 minisilos (unidades experimentales) con capacidad de $5.0 \mathrm{~kg}$, utilizando $1.5 \mathrm{~kg}$ para los análisis de laboratorio. El forraje fue compactado con el propósito de eliminar el aire del interior de los minisilos y se almacenaron en los ambientes del LUNEA, en posición vertical por 21 y 42 días de fermentación. Luego se analizaron y se procesaron para determinar la composición química bromatológica. Se utilizaron las técnicas descritas en AOAC (1990) para determinar materia seca (MS) y materia mineral (MM), y el método Dumas para determinar proteína cruda (PC), y Van Soest (1994) para determinar fibra detergente neutro (FDN) y fibra detergente ácida (FDA).

Para el experimento se empleó un diseño estadístico completamente al azar en un arreglo factorial de $2 \times 2 \times 4$. Se evaluaron dos especies de ensilado (avena y cebada) en dos tiempos de fermentación ( 21 y 42 días) con cuatro niveles de levadura $S$. cerevisiae ( 0 , 15,30 y $45 \mathrm{~g} / \mathrm{kg} \mathrm{FV}$ ), con tres repeticiones por tratamiento. El modelo estadístico fue: $\mathrm{Y}_{\mathrm{ij \textrm {jl }}}$ $=\mu+\mathrm{E}_{\mathrm{i}}+\mathrm{T}_{\mathrm{j}}+\mathrm{N}_{\mathrm{k}}+\mathrm{ET}_{\mathrm{ij}}+\mathrm{EN}_{\mathrm{ik}}+\mathrm{TN}_{\mathrm{jk}}+$ $\mathrm{ETN}_{\mathrm{ijk}}+\mathrm{e}_{\mathrm{ijk},}$, donde: $\mathrm{Y}_{\mathrm{ijkl}}=$ variable respuesta (MS, PC, MM, EE, FDN, FDA expresados en porcentaje y valor de $\mathrm{pH}) ; \mu=$ Media general; $\mathrm{E}_{\mathrm{i}}=$ Efecto del i- ésimo nivel del factor Especie; $\mathrm{T}_{\mathrm{j}}=$ Efecto del $\mathrm{j}$ - ésimo nivel del factor Tiempos de fermentación; $\mathrm{N}_{\mathrm{k}}=$ Efecto del k- ésimo nivel del factor Nivel de levadura; $\mathrm{ET}_{\mathrm{ij}}=$ Efecto del i-ésimo nivel del factor de la interacción especie*tiempo de fermentación; $\mathrm{EN}_{\mathrm{ik}}=$ Efecto del i-ésimo nivel del factor de interacción Especie*Nivel de levadura; $\mathrm{TN}_{\mathrm{jk}}=$ Efecto del $\mathrm{j}$-ésimo nivel del factor de la interacción tiempo de fermentación*nivel de levadura; ETN $_{\mathrm{ijk}}=$ Efecto del i-ésimo nivel del factor de interacción ntiempo de fermentación*Nivel de levadura; $\mathrm{y}_{\mathrm{ijkl}}=$ Error experimental.

Para el procesamiento y análisis de datos se utilizó el programa estadístico InfoStat v. 2020, mediante un análisis de varianza y la prueba de comparación de medias Tukey a la probabilidad de $5 \%$. 


\section{Resultados y Discusión}

Los resultados de las variables del ensilado de avena y cebada forrajera con cuatro niveles de $S$. cerevisiae y 2 tiempos de fermentación, así como la estimación de los coeficientes de regresión del nivel de levadura se presentan en el Cuadro 1.

El factor ensilado y el tiempo de fermentación tuvo un efecto significativo $(p<0.05)$ sobre las variables estudiadas salvo para EE y PC, en tanto que el factor nivel de levadura tuvo un efecto significativo $(\mathrm{p}<0.05)$ sobre las variables excepto para EE. La interacción ensilado*tiempo mostró un efecto significativo $(\mathrm{p}<0.05)$ sobre la MS, FDN y FDA, pero no sobre PC, EE, MM y $\mathrm{pH}$. La interacción ensilado*nivel tuvo un efecto significativo $(\mathrm{p}<0.05)$ sobre las variables estudiadas excepto para PC, EE, MM y $\mathrm{pH}$. La interacción tiempo*nivel solo tuvo un efecto significativo $(p<0.05)$ sobre MM, y la interacción ensilado*tiempo de fermentación*nivel de levadura no presentó un efecto significativo sobre las variables estudiadas $(\mathrm{p}>0.05)$ (Cuadro 1).

El contenido de MS para la avena fue de 23.91 y $22.62 \%$ a los 21 y 42 días $\sin S$. cerevisiae. En el caso de la cebada fue de $20.30 \% \sin S$. cerevisiae a los 21 días y $19.11 \%$ con $S$. cerevisiae de $45 \mathrm{~g} / \mathrm{kg} \mathrm{FV} \mathrm{a}$ los 42 días de fermentación. Zhou et al. (2019) reportó en ensilado de tallos de maíz a los 30 días de fermentación con promedios similares de $26.9 \%$ MS sin S. cerevisiae y $26.0 \%$ MS con $S$. cerevisiae a los 30 días de fermentación. Por otro lado, Duniere et al. (2015) en ensilado de maíz en 90 días de fermentación con $S$. cerevisiae reportaron promedios mayores de $31.40 \% \mathrm{MS}$, en tanto que Kamphayae et al. (2016) en ensilado de paja de arroz con pulpa de yuca obtuvieron $39.0 \%$ MS con adición de $S$. cerevisiae a las ocho semanas de almacenamiento. Los resultados observados en la presente investigación pueden deberse a que ambas gramíneas realizaron una fermentación completa durante los 21 y 42 días de almacenamiento.
Los mejores valores de PC en la avena se observaron a los 21 y 42 días (17.06 y $16.81 \%$ ) y en el caso de la cebada fueron de 17.98 y $17.11 \%$ a los 21 días y 42 días de fermentación con $S$. cerevisiae de $45 \mathrm{~g} / \mathrm{kg}$ $\mathrm{FV}$, respectivamente. Estos resultados superan los reportados por Zhou et al. (2019) en ensilado de tallos de maíz a los 30 y 60 días de fermentación con S. cerevisiae (PC 8.5 y 8\%), a los de Duniere et al. (2015) en ensilado de maíz en 90 días de fermentación (PC 9.719.91\%), de Ok et al. (2006) en ensilado de paja de arroz a los 20 días de fermentación (PC 8.37\%) y de Saeed et al. (2017) en ensilado de residuos de cultivos de maíz a los 60 días con 10 g. S. cerevisiae (PC 5.47\%), pero similares a los de Contreras et al. (2020) en ensilado de cebada a los 24 días de fermentación con 10 y $15 \mathrm{~g} / \mathrm{kg}$ de $S$. cerevisiae (PC 15.8 y $16.2 \%$, respectivamente). El incremento del porcentaje de $\mathrm{PC}$ al adicionar $S$. cerevisiae posiblemente se deba a que la proteína se deriva no solo de las proteínas vegetales sino además de las proteínas microbianas, especialmente de células de $S$. cerevisiae que contienen entre 40 y $60 \%$ de proteína (Zhou et al., 2019).

Los mayores valores de FDN se presentaron en la avena con $S$. cerevisiae de 15 $\mathrm{g} / \mathrm{kg}$ FV a los 42 días (52.69\%) y con $S$. cerevisiae de $30 \mathrm{~g} / \mathrm{kg} \mathrm{FV}$ a los 42 días $(52.36 \%)$, en tanto que en la cebada fue en el tratamiento $\sin S$. cerevisiae $(54.66 \%)$ y con $S$. cerevisiae de $15 \mathrm{~g} / \mathrm{kg} \mathrm{FV}$ a los 21 días $(52.86 \%)$. En este sentido, los resultados estuvieron en los rangos obtenidos por Duniere et al. (2015); Kamphayae et al. (2016), Zhou et al. (2019) y Contreras et al. (2020) con ensilados de diversos forrajes utilizando $S$. cerevisiae como aditivo.

Los mejores valores de FDA de la avena se observaron en el ensilado con $S$. cerevisiae de $15 \mathrm{~g} / \mathrm{kg} \mathrm{FV}$ a los 42 días (29.99\%) y con $S$. cerevisiae de $30 \mathrm{~g} / \mathrm{kg} \mathrm{FV}$ a los 42 días de fermentación (30.01\%) y en la cebada $\sin S$. cerevisiae a $\operatorname{los} 21$ días $(33.02 \%)$ y con $S$. cerevisiae de $15 \mathrm{~g} / \mathrm{kg} \mathrm{FV}$ a los 21 días (31.90\%). Zhou et al. (2019) 
Cuadro 1. Composición química bromatológica del ensilado de avena y cebada forrajera con diferentes niveles de Saccharomyces cerevisiae en dos tiempos de fermentación

\begin{tabular}{|c|c|c|c|c|c|c|c|c|c|}
\hline 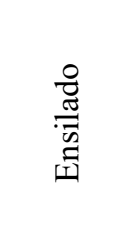 & 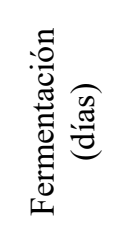 & 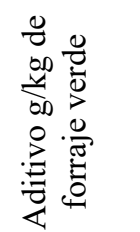 & MS\% & $\mathrm{PC} \%$ & MM\% & FDN\% & FDA $\%$ & $\mathrm{EE} \%$ & $\mathrm{pH}$ \\
\hline \multirow[t]{8}{*}{ Avena } & 21 & 0 & $23.91^{\mathrm{a}}$ & $8.86^{\mathrm{d}}$ & $4.00^{c}$ & $48.66^{\mathrm{abc}}$ & $27.13^{\mathrm{ab}}$ & $3.82^{\mathrm{a}}$ & $4.38^{\mathrm{b}}$ \\
\hline & & 15 & $21.09^{\text {abcde }}$ & $11.8^{\mathrm{cd}}$ & $4.48^{c}$ & $51.52^{a b c}$ & $28.45^{\mathrm{ab}}$ & $4.46^{\mathrm{a}}$ & $5.34^{\mathrm{ab}}$ \\
\hline & & 30 & $21.59^{\mathrm{abcd}}$ & $13.21^{\mathrm{abcd}}$ & $4.74^{\mathrm{c}}$ & $52.13^{\mathrm{ab}}$ & $29.17^{\mathrm{ab}}$ & $3.79^{\mathrm{a}}$ & $5.22^{\mathrm{ab}}$ \\
\hline & & 45 & $22.28^{\mathrm{ab}}$ & $17.06^{\mathrm{ab}}$ & $4.78^{c}$ & $49.30^{\mathrm{abc}}$ & $27.28^{\mathrm{ab}}$ & $4.13^{\mathrm{a}}$ & $5.30^{\mathrm{ab}}$ \\
\hline & 42 & 0 & $22.62^{\mathrm{ab}}$ & $9.17^{\mathrm{d}}$ & $4.01^{\mathrm{c}}$ & $46.80^{\mathrm{abc}}$ & $26.05^{b}$ & $3.31^{\mathrm{a}}$ & $4.32^{\mathrm{b}}$ \\
\hline & & 15 & $21.95^{\mathrm{abc}}$ & $15.07^{\mathrm{abc}}$ & $4.24^{\mathrm{c}}$ & $52.69^{\mathrm{a}}$ & $29.99^{\mathrm{ab}}$ & $3.51^{\mathrm{a}}$ & $4.88^{\mathrm{ab}}$ \\
\hline & & 30 & $20.98^{\text {bcde }}$ & $14.54^{\mathrm{abc}}$ & $4.79^{c}$ & $52.36^{\mathrm{a}}$ & $30.01^{\mathrm{ab}}$ & $3.49^{\mathrm{a}}$ & $4.88^{\mathrm{ab}}$ \\
\hline & & 45 & $21.62^{\mathrm{abcd}}$ & $16.81^{\mathrm{ab}}$ & $4.80^{c}$ & $48.17^{\mathrm{abc}}$ & $27.03^{\mathrm{ab}}$ & $3.58^{\mathrm{a}}$ & $4.91^{\mathrm{ab}}$ \\
\hline \multirow[t]{9}{*}{ Cebada } & 21 & 0 & $20.30^{\text {bcded }}$ & $9.13^{\mathrm{d}}$ & $8.25^{\mathrm{b}}$ & $54.66^{\mathrm{a}}$ & $33.02^{\mathrm{a}}$ & $3.17^{\mathrm{a}}$ & $4.88^{\mathrm{ab}}$ \\
\hline & & 15 & $18.73^{\mathrm{def}}$ & $12.56^{\mathrm{bcd}}$ & $9.47^{\mathrm{ab}}$ & $52.86^{\mathrm{a}}$ & $31.90^{\mathrm{ab}}$ & $3.32^{\mathrm{a}}$ & $5.45^{\mathrm{ab}}$ \\
\hline & & 30 & $20.17^{\text {bcdef }}$ & $15.54^{\mathrm{abc}}$ & $8.93^{\mathrm{ab}}$ & $47.70^{\mathrm{abc}}$ & $28.28^{\mathrm{ab}}$ & $3.39^{\mathrm{a}}$ & $5.97^{\mathrm{a}}$ \\
\hline & & 45 & $21.57^{\mathrm{abcd}}$ & $17.98^{\mathrm{a}}$ & $8.74^{\mathrm{ab}}$ & $47.40^{\mathrm{abc}}$ & $28.70^{\mathrm{ab}}$ & $2.91^{\mathrm{a}}$ & $6.22^{\mathrm{a}}$ \\
\hline & 42 & 0 & $17.67^{\mathrm{f}}$ & $9.56^{d}$ & $9.31^{\mathrm{ab}}$ & $48.65^{\mathrm{abc}}$ & $29.25^{\mathrm{ab}}$ & $3.78^{\mathrm{a}}$ & $5.38^{\mathrm{ab}}$ \\
\hline & & 15 & $18.42^{\mathrm{ef}}$ & $12.59^{\mathrm{bcd}}$ & $8.64^{\mathrm{ab}}$ & $47.65^{\mathrm{abc}}$ & $28.48^{\mathrm{ab}}$ & $2.70^{\mathrm{a}}$ & $4.78^{\mathrm{ab}}$ \\
\hline & & 30 & $17.63^{\mathrm{f}}$ & $14.76^{\mathrm{abc}}$ & $9.49^{\mathrm{a}}$ & $44.06^{\mathrm{bc}}$ & $26.43^{b}$ & $4.13^{\mathrm{a}}$ & $5.15^{\mathrm{ab}}$ \\
\hline & & 45 & $19.11^{\text {cdef }}$ & $17.11^{\mathrm{ab}}$ & $9.07^{\mathrm{ab}}$ & $43.61^{c}$ & $26.15^{\mathrm{b}}$ & $3.02^{\mathrm{a}}$ & $5.52^{\mathrm{ab}}$ \\
\hline & SEM & & 0.46 & 0.78 & 0.59 & 0.8 & 0.5 & 0.12 & 0.13 \\
\hline \multirow[t]{7}{*}{ P- value } & Ensilad & & $<0.01$ & 0.44 & $<0.01$ & 0.02 & 0.11 & 0.08 & $<0.01$ \\
\hline & Nivel & & $<0.01$ & $<0.01$ & $<0.01$ & 0.01 & 0.04 & 0.85 & $<0.01$ \\
\hline & Ensilad & o*Nivel & 0.01 & 0.42 & 0.17 & $<0.01$ & $<0.01$ & 0.31 & 0.28 \\
\hline & Tiempo & & $<0.01$ & 0.33 & 0.31 & $<0.01$ & 0.02 & 0.46 & 0.01 \\
\hline & Ensilad & o*Tiempo & $<0.01$ & 0.11 & 0.17 & 0.01 & 0.01 & 0.12 & 0.71 \\
\hline & Nivel*' & iempo & 0.06 & 0.40 & 0.04 & 0.69 & 0.60 & 0.54 & 0.15 \\
\hline & $\begin{array}{l}\text { Ensilad } \\
\text { Tiempo }\end{array}$ & o*Nivel* & 0.96 & 0.55 & 0.16 & 0.85 & 0.83 & 0.94 & 0.56 \\
\hline \multirow[t]{2}{*}{ Nivel } & Linear & & 0.93 & $<0.01$ & $<0.01$ & $<0.01$ & 0.02 & 0.91 & $<0.01$ \\
\hline & Cuadrá & ica & 0.93 & 0.22 & 0.06 & 0.03 & 0.07 & 0.59 & 0.51 \\
\hline
\end{tabular}

MS, materia seca; PC, proteína cruda; MM, materia mineral; FDN, fibra detergente neutra; FDA, fibra detergente ácida; $\mathrm{pH} ; 3 \mathrm{SEM}$, error estándar de la media

a-f Medias seguidas con diferentes superíndices en una misma columna no difieren entre sí $(p<0.05)$ 
encontraron valores mayores en ensilado de tallos de maíz de $43 \%$ FDA con $S$. cerevisiae a los 30 días. Valores ligeramente superiores fueron obtenidos por Contreras et al. (2020) en ensilado de cebada a los 24 días de fermentación con $10 \mathrm{~g} / \mathrm{kg}$ de $S$. cerevisiae (33.1\%) FDA y con $15 \mathrm{~g} / \mathrm{kg}$ de $S$. cerevisiae (37.0\%), en tanto que Duniere et al. (2015) con ensilado de maíz a 90 días de fermentación reportaron promedios ligeramente menores $(27.23 \%)$.

El ligero aumento de FDN y FDA en los ensilajes con $S$. cerevisiae podría atribuirse en gran parte a la disminución relativa de los carbohidratos fermentables, incluyendo los carbohidratos solubles en agua, el almidón y la pérdida de MS (Zhou et al., 2019). La pared celular de las plantas es la fracción insoluble del forraje después de exponerlo al tratamiento con solución detergente neutro. Esta fracción contiene celulosa, hemicelulosa, sílice, proteína indigestible y lignina (Van Soest et al., 1991). De acuerdo con Van Soest et al. (1994), los forrajes con un contenido menor a $40 \%$ de FDN pueden considerarse como de buena calidad.

Los mejores contenidos de EE en ensilado de avena fueron los tratamientos con $S$. cerevisiae de 15 y $45 \mathrm{~g} / \mathrm{kg} \mathrm{FV}$ a los 21 y 42 días (4.46 y 4.13\%), respectivamente, y de cebada en aquellos $\sin S$. cerevisiae a los 42 días (3.78\%) y con S. cerevisiae de $30 \mathrm{~g} / \mathrm{kg}$ FV a los 42 días (4.13\%). Estos resultados fueron similares a los obtenidos por Duniere et al. (2015) con ensilado de maíz con $S$. cerevisiae a los 90 días, y a los de Contreras et al. (2020) en ensilado de cebada a los 24 días de fermentación con 10 y $15 \mathrm{~g} / \mathrm{kg}$ de $S$. cerevisiae. No obstante, superiores a los valores reportados por Kamphayae et al. (2016) en ensilado de paja de arroz con pulpa de yuca con adición de 20 y $35 \%$ de $S$. cerevisiae $(1.15$ y $1.22 \%)$, respectivamente a las ocho semanas de almacenamiento. El incremento en el contenido de extracto etéreo, según Saeed et al. (2017) pudo deberse a la formación de ácidos grasos volátiles (AGV) durante el proceso de ensilado.
Con relación al contenido de materia mineral, el mejor valor se obtuvo para la avena con 4.78 y $4.80 \%$ de MM con $S$. cerevisiae de $45 \mathrm{~g} / \mathrm{kg} \mathrm{FV}$ a los 21 y 42 días de fermentación, respectivamente y para la cebada de $9.47 \%$ MM con $S$. cerevisiae de $15 \mathrm{~g} / \mathrm{kg}$ FV a los 21 días y $9.49 \% \mathrm{MM}$ con $S$. cerevisiae de $30 \mathrm{~g} / \mathrm{kg} \mathrm{FV}$ a los 42 días de fermentación. En este sentido, Poma (2011) reportó que con la adición de $10 \% \mathrm{~S}$. cerevisiae en ensilado de cebada se encontró 7.28\% de MM en 90 días de fermentación, valores ligeramente inferiores a los de la presente investigación; en tanto que Leao et al. (2017) con 60 días de fermentación sin $S$. cerevisiae. reportaron $5.0 \%$ de MM. Según Poma (2011) el aumento considerable del contenido de minerales en el ensilado probablemente sea debido al estado productivo lechoso y a la contaminación del suelo ( $>10 \%)$ (Acosta, 2002).

Los mayores valores de $\mathrm{pH}$ se observaron en los tratamientos en avena de 5.34 con $S$. cerevisiae de $15 \mathrm{~g} / \mathrm{kg} \mathrm{FV}$ a los 21 días y $5.30 \mathrm{pH}$ con $S$. cerevisiae de $45 \mathrm{~g} / \mathrm{kg} \mathrm{FV} \mathrm{a}$ los 21 días; en tanto que la cebada fue de 6.22 y 5.52 con $S$. cerevisiae de $45 \mathrm{~g} / \mathrm{kg} \mathrm{FV}$ en 21 y 42 días de fermentación, respectivamente. Zhou et al. (2019) reportaron en ensilado de tallos de maíz 4.42 de $\mathrm{pH}$ a los 30 días y 4.45 a los 60 días con $S$. cerevisiae y 3.57 y 3.66 de $\mathrm{pH}$ en 30 y 60 días, respectivamente, $\sin S$. cerevisiae, valores inferiores a los obtenidos en la presente investigación. Por otro lado, Ok et al. (2006) encontró 4.13 de $\mathrm{pH}$ en ensilado de paja de arroz a los 20 días de fermentación con $S$. cerevisiae, en tanto que Kamphayae et al. (2016) en ensilado de paja de arroz con pulpa de yuca obtuvieron 4.29 de $\mathrm{pH}$ con adición del $20 \%$ S. cerevisiae y 4.51 de $\mathrm{pH}$ con $35 \% S$. cerevisiae entre las 0 y 8 semanas de almacenamiento.

Los resultados obtenidos en la presente investigación difieren con lo manifestado por Maynard y Loosly (1992), quienes indican que el pH debe estar en 4.5 para un buen ensilado, evitando la putrefacción. El pH como variable sirve como indicador de la calidad 
fermentativa (Jobim et al., 2007). Mayores niveles de $\mathrm{pH}$ pueden provocar rechazo durante el consumo y no permite conservar bien la calidad del ensilaje. Una de las causas por la cual incrementó el contenido de $\mathrm{pH}$ es que en la fase aeróbica no se extrajo todo el oxígeno presente en la masa ensilada. La exposición al oxígeno promueve el crecimiento de levaduras y el consumo de ácido láctico, aumentando el $\mathrm{pH}$ del ensilaje y permitiendo la proliferación de microbios menos tolerantes a los ácidos, como los mohos. Debido a esta correlación, a menudo se considera que las levaduras contribuyen al deterioro aeróbico del ensilaje (Woolford, 1990), ya sea durante la fase aeróbica antes del ensilado o durante la alimentación (Driehuis y Elferink, 2000).

\section{Conclusiones}

- La composición química del ensilado de avena con adición de levadura $S$. cerevisiae de $45 \mathrm{~g} / \mathrm{kg} \mathrm{FV}$ a los 42 días de tiempo de fermentación muestra mejores resultados.

- La adicción de mayores niveles de Saccharomyces cerevisiae en el ensilado de avena y cebada causó un gran incremento en el contenido de proteína y ligeros incrementos en extracto etéreo y materia mineral.

- El pH de los ensilados de avena y cebada en los diferentes niveles y tiempos de cosecha fueron en rango de 4.78 a 6.22.

\section{Literatura Citada}

1. Acosta Y. 2002. Ensilajes de pasturas: algunas consideraciones para su confección. Uruguay: Unidad de Agronegocios y Difusión del INIA. 13 p.

2. [AOAC] Association of Official Analytical Chemists. 1990. Official method of analysis. USA: AOAC.

3. Broadway PR, Carroll JA, Sanchez $N C$. 2015. Live yeast and yeast cell wall supplements enhance immune function and performance in food-producing livestock: a review. Microorganisms 3: 417-427. doi: 10.3390/microorganisms3030417

4. Contreras JL, Quichca RG, Cordero AG, Rojas YC, Curasma J. 2020. Nutritive value of barley silage (Hordeum vulgare $\mathrm{L}$ ) with different levels of Saccharomyces cerevisiae at different ensiling lengths. Iraqi J Agric Sci 51: 1350-1356. doi: 10.36103/ijas.v51i5.1144

5. Duniere L, Jin L, Smiley B, Qi M, Rutherford W, Wang Y, McAllister T. 2015. Impact of adding Saccharomyces strains on fermentation, aerobic stability, nutritive value, and select Lactobacilli populations in corn silage. J Anim Sci 93: 2322-2335. doi: 10.2527/jas.2014-8287

6. Driehuis F, Elferink SJ. 2000. The impact of the quality of silage on animal health and food safety: a review. Vet Quart 22: 212-216. doi: 10.1080/01652176-2000.9695061

7. Elghandour MMY, Mellado M, Kholif $A E$, Salem AZM, Barbabosa A, Ballinas S, Esquivel A, et al. 2016. Fecal gas production of ten common horse feeds supplemented with Saccharomyces cerevisiae. J Equine Vet Sci 47: 1-8. doi: 10.1016/j.jevs.2016.07.008

8. Jobim CC, Nussio LG, Reis RA, Schmidt P. 2007. Avanços metodológicos na avaliação da qualidade da forragem conservada. Rev Bras Zootecn 36: 101-119. doi: 10.1590/s151635982007001000013

9. Kamphayae S, Kumagai H, Bureenok S, Narmseelee R, Butcha P. 2016. Effects of graded levels of liquid brewer's yeast on chemical composition and fermentation quality in cassava pulp and rice straw-based total mixed ration silage. Anim Sci J 88: 618-624. doi: 10.1111/ asj. 12682

10. Leao GFM, Jobim CC, Neumann M, Horst EH, Santos SKD, Venancio BJ, Santos LC. 2017. Nutritional composition and aerobic stability of winter cereal silage at different storage times. Acta Sci Anim Sci 39(2). doi: 10.4025/ actascianimsci.v39i2.34270 
11. McAllister TA, Beauchemin KA, Alazzeh AY, Baah J, Teather. RM, Stanford K. 2011. The use of direct fed microbials to mitigate pathogens and enhance production in cattle. Can J Anim Sci 91: 193-211. doi:10.4141/CJAS10047

12. Maynard LA, Loosli JK. 1992. Nutrición animal. McGraw-Hill. 640 p.

13. Muck RE, Nadeau EMG, McAllister TA, Contreras-Goeva FE, Santos MC, Kung L. 2018. Silage review. Recent advances and future uses of silage additives. J Dairy Sci 101: 3980-4000. doi: 10.3168/jds.2017-13839

14. Ok JU, Lee SM, Lee SJ, Lim JH, Kang TW, Jung HY, Moon YH, Lee SS. 2006. Effect of yeast addition in rice straw silage fermentation. J Anim Sci Technol 48:691-698. doi: 10.5187/jast.2006-48.5.691

15. Ordoñez J, Bojórquez C. 2011. Мanejo del establecimiento de pasturas para zonas altoandinas del Perú. Lima, Perú: CONCYTEC. $391 \mathrm{p}$.

16. Poma C. 2011. Evaluación del efecto de diferentes aditivos en la composición química del ensilaje de cebada (Hordeum vulgare) para la alimentación del ganado en el municipio de Viacha. Tesis de Ingeniero Agrónomo. La Paz, Bolivia: Univ. Mayor de San Andrés. 84 p.

17. Roque B. 2012. Nutrición animal. Puno, Perú: Univ. Nacional del Altiplano Puno. $198 \mathrm{p}$.

18. Saeed A, Al Sultani H, Mottaleb A. 2017. Effect of addition of different levels of baker's yeast Saccharomyces cerevisae on fermentation quality and nutritive value of ensiled corn crop residuals. Euphrates J Agric Sci 9: 8-18.

19. Shurson GC. 2018. Yeast and yeast derivatives in feed additives and ingredients: sources, characteristics, animal responses, and quantification methods. Anim Feed Sci Technol 235: 60-76. doi: 10.1016/j.anifeedsci.2017.11.010

20. Trckova M, Faldyna M, Alexa P, Zajacova ZS, Gopfert E, Kumprechtova D, Auclair E, et al. 2014. The effects of live yeast Saccharomyces cerevisiae on postweaning diarrhea, immune response, and growth performance in weaned piglets. J Anim Sci 92: 767-774. doi: 10.2527/jas.20136793

21. Van Soest PVJ. 1994. Nutritional ecology of the ruminant. $2^{\text {nd }}$ ed. Cornell University Press. 488 p.

22. Van Soest PJ, Robertson JB, Lewis BA. 1991. Methods for dietary fiber, neutral detergent fiber, and nonstarch polysaccharides in relation to animal nutrition. $\mathrm{J}$ Dairy Sci 74: 3583-3597. doi: 10.3168/ jds.s0022-0302(91)78551-2

23. Woolford MK. 1990. The detrimental effects of air on silage. J Appl Bacteriol 68: 101-116. doi: 10.1111/j.1365-2672.1990.tb02554.x

24. Zhu W, Wei Z, Xu N, Yang F, Yoon I, Chung Y, Liu J, Wang J. 2017. Effects of Saccharomyces cerevisiae fermentation products on performance and rumen fermentation and microbiota in dairy cows fed a diet containing low quality forage. J Anim Sci Biotechnol 8: 36. doi: 10.1186/s40104-017-0167-3

25. Zhou X, Ouyang Z, Zhang X, Wei Y, Tang S, Ma Z, Tan Z, Zhu N, Teklebrhan T, Han X. 2019. Sweet Corn stalk treated with Saccharomyces cerevisiae alone or in combination with lactobacillus plantarum: nutritional composition, fermentation traits and aerobic stability. Animals 9: 598. doi: 10.3390/ani9090598 